\title{
A!
}

This is an electronic reprint of the original article.

This reprint may differ from the original in pagination and typographic detail.

Saloriutta, K.; Hancock, Y.; Kärkkäinen, A.; Kärkkäinen, Leo; Puska, M.J.; Jauho, A.P.

\section{Electron Transport in Edge-Disordered Graphene Nanoribbons}

Published in:

Physical Review B

DOI:

10.1103/PhysRevB.83.205125

Published: 23/05/2011

Document Version

Publisher's PDF, also known as Version of record

Please cite the original version:

Saloriutta, K., Hancock, Y., Kärkkäinen, A., Kärkkäinen, L., Puska, M. J., \& Jauho, A. P. (2011). Electron

Transport in Edge-Disordered Graphene Nanoribbons. Physical Review B, 83(20), 1-6. [205125].

https://doi.org/10.1103/PhysRevB.83.205125

This material is protected by copyright and other intellectual property rights, and duplication or sale of all or part of any of the repository collections is not permitted, except that material may be duplicated by you for your research use or educational purposes in electronic or print form. You must obtain permission for any other use. Electronic or print copies may not be offered, whether for sale or otherwise to anyone who is not an authorised user. 


\title{
Electron transport in edge-disordered graphene nanoribbons
}

\author{
Karri Saloriutta, ${ }^{1, *}$ Y. Hancock, ${ }^{2}$ Asta Kärkkäinen, ${ }^{3}$ Leo Kärkkäinen, ${ }^{3}$ Martti J. Puska, ${ }^{1}$ and Antti-Pekka Jauho ${ }^{1,4}$ \\ ${ }^{1}$ Department of Applied Physics, Aalto University School of Science, P.O. Box 11100, FIN-00076 AALTO, Finland \\ ${ }^{2}$ Department of Physics, The University of York, Heslington, York YO10 5DD, United Kingdom \\ ${ }^{3}$ Nokia Research Center, FIN-00180 Helsinki, Finland \\ ${ }^{4}$ DTU Nanotech, Department of Micro- and Nanotechnology, Technical University of Denmark, Kongens Lyngby DK-2800, Denmark
}

(Received 20 August 2010; revised manuscript received 25 February 2011; published 23 May 2011)

\begin{abstract}
$A b$ initio methods are used to study the spin-resolved transport properties of graphene nanoribbons (GNRs) that have both chemical and structural edge disorder. Oxygen edge adsorbates on ideal and protruded ribbons are chosen as representative examples, with the protrusions forming the smallest possible structural disorder consistent with the edge geometry. The impact of the oxygen adsorbate dominates the transport properties of armchair nanoribbons. For zigzag nanoribbons, the transmission properties are markedly affected by the protrusion alone, leading to spin-polarized transport and a smaller perturbation from the oxygen adsorbate. Armchair nanoribbons also exhibit, as a function of their width and the threefold family structure, a repeating pattern related to the existence of the spin polarization and to the variation in the width of the band gap.
\end{abstract}

DOI: 10.1103/PhysRevB.83.205125

PACS number(s): 71.15.Mb, 72.80.Vp, 75.75.-c, 73.22.Pr

\section{INTRODUCTION}

After Novoselov et al. demonstrated that single layer graphite (i.e., graphene) can be produced using a simple experimental procedure, ${ }^{1}$ interest in the properties of this new material has exploded. Of particular promise are graphene nanoribbons (GNRs) that are made by cutting the graphene sheet into ribbons of finite widths. GNRs have band gaps that scale inversely against their width. ${ }^{2}$ Their semiconducting character and tuneable band gap may enable the use of GNRs in future post-Moore electronics as field-effect transistors, ${ }^{3}$ chemical sensors, ${ }^{4}$ or spintronic devices. ${ }^{5}$ Several methods for fabricating GNRs have been demonstrated: lithographic patterning, ${ }^{2,6}$ chemical means, ${ }^{7,8}$ and most recently by opening carbon nanotubes. ${ }^{9}, 10$ The lithographic methods suffer from difficulty in controlling the crystallographic orientation and smoothness of the ribbons edge, whereas chemical methods ${ }^{8}$ and annealing the unzipped nanotubes ${ }^{11}$ produce better results in these respects. Scanning tunneling microscopy (STM) indicates that armchair is the most stable edge type, ${ }^{12}$ while recent experiments show that the zigzag edge is preferred when edge reconstruction occurs. ${ }^{13,14}$ This apparent contradiction can be understood by the kinetics of the edge atoms ${ }^{15}$ and indicates that there are different possibilities for microscopic control of graphene edges.

Fujita et al. ${ }^{16}$ used a tight-binding model to predict the existence of localized (flat-band) edge states for hydrogenpassivated zigzag edges, which were later confirmed by DFT calculations. ${ }^{17}$ The edge states in the zigzag GNRs (ZGNRs) lead to magnetic structures which are ferromagnetic along the ribbons and antiferromagnetic across the ribbons. Although the edge states have been seen in STM experiments, ${ }^{12}$ we are not aware of any evidence for magnetic edge ordering. STM and DFT calculations do agree on the energetic favorability of the armchair edge when compared to the zigzag edge. ${ }^{18}$ Recent DFT calculations also indicate the possibility of a zigzag edge reconstruction that leads to a new stable edge type, albeit without the magnetization. ${ }^{19}$ Stability of the zigzag edge may also be gained through nonideal hydrogen passivation, which also suppresses the spin polarization. ${ }^{20}$
Transport experiments on GNRs demonstrate the crucial role that disorder (particularly edge disorder) plays. ${ }^{21,22}$ Theory predicts edge disorder to manifest as a widening of the transport gap and overall suppression of the conductance through Anderson localization, ${ }^{23}$ or by the Coulomb blockade effect for edges that are heavily disordered. ${ }^{24}$ Most of the theoretical work, to date, has been based on a tight-binding description, which remains computationally tractable for large, experimental-sized systems, compared to more computationally demanding ab initio approaches.

Although a tight-binding level treatment is often sufficiently accurate for dealing with edge disorder, especially when relaxation and spin-polarization effects are accounted for, ${ }^{17,25}$ this picture changes when the role of chemical disorder is considered. Chemical dopants often have complicated interactions with the graphene edge involving charge transfer and magnetic effects. Dopants can thus have a large impact on both the electronic ${ }^{26,27}$ and transport ${ }^{28}$ properties of ideal nanoribbons and an ab initio approach is generally needed. The interplay between structural and chemical disorders presents a complicated and rather unexplored domain and the first reports are just beginning to emerge (see, e.g., Ref. 25).

\section{SYSTEMS AND METHODS}

In this work, $a b$ initio methods ${ }^{29,30}$ are used to study transport properties of GNRs that have small protrusions and edge adsorbates. We restrict ourselves to oxygen as the adsorbate due to its common use in many of the processes for synthesizing GNRs, such as oxygen plasma etching, or the breaking of carbon-carbon bonds with oxygenating chemical reactions. ${ }^{10}$ Our main focus is on the interplay between the two types of disorder caused by the adsorbate and by the inhomogeneous edge, and how this affects transmission properties of nanoribbons. In contrast with a recent work exploring similar issues, ${ }^{25}$ we especially study spin-polarization and charge transfer effects between the ribbon and the adsorbate and the resultant spin-polarized transport.

Figure 1 shows the representative systems under study. Two semi-infinite leads formed by ideal hydrogen-passivated GNRs 

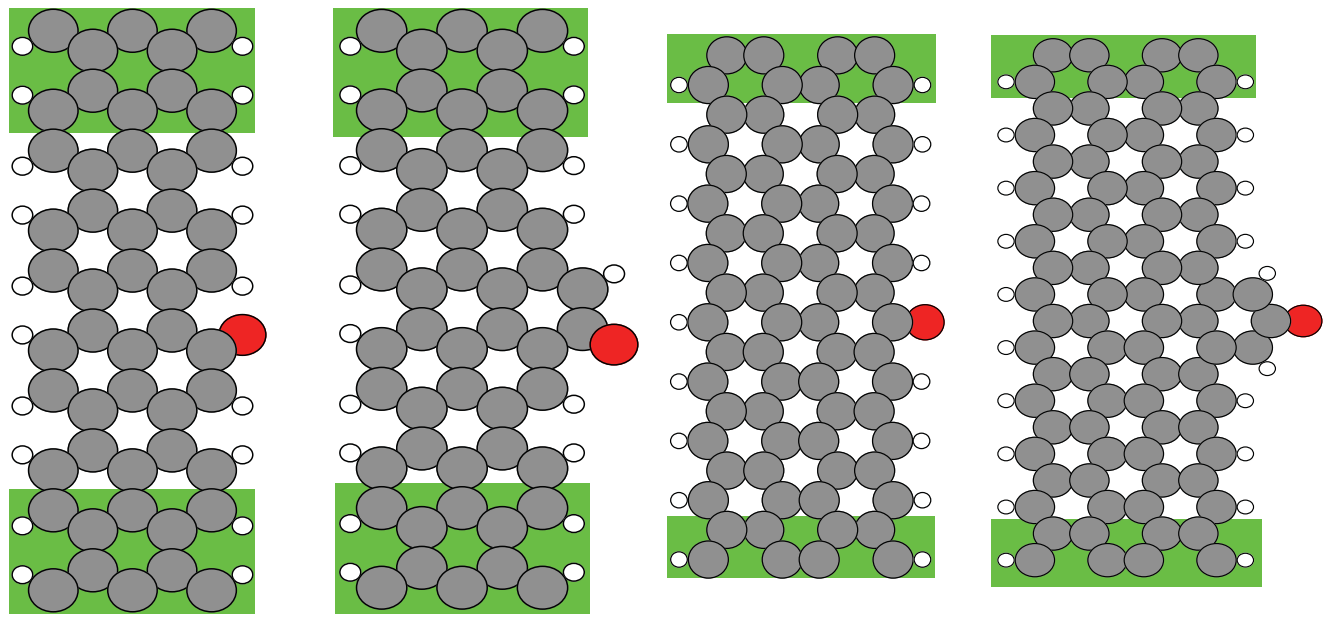

FIG. 1. (Color online) Schematics of the representative two-probe transport systems under study. (From left to right) An ideal 5-AGNR, a 5-AGNR with a protrusion, an ideal 4-ZGNR, and a 4-ZGNR with a protrusion. Each system contains one adsorbed oxygen atom. The (green) background-shaded areas show the first principal layers of the semi-infinite leads. Gray, dark (red), and white circles indicate carbon, oxygen, and hydrogen atoms, respectively.

of either armchair GNR (AGNR) or zigzag (ZGNR) type are connected by a central device region of the same width, which consists of an ideal or protruded GNR with an adsorbed oxygen atom. We calculate the coherent transmission of electrons through these systems to study the scattering properties of the oxygen and the protrusions and compare these results to systems passivated by hydrogen only.

We relax the ionic coordinates using a conjugate gradient algorithm until the force on each atom is within $0.02 \mathrm{eV} / \AA$. A double- $\zeta$ polarization (DZP) basis set is used together with a 250 Ry mesh cutoff, and the PBE-GGA functional ${ }^{31}$ is used for electron exchange and correlation. In addition to studying the transport properties of these systems, we also investigate the behavior of the bond dissociation energy (BDE) of the oxygen atom. The BDE is calculated by subtracting the energy of the relaxed total system (ribbon and oxygen) from the sum of the energies of the constituent parts (ribbon without oxygen and a single oxygen atom) obtained by using the atomic coordinates of the relaxed full system and the same computational cell and parameters. Basis-set-superposition errors are corrected for by using the counterpoise correction. ${ }^{32}$

Transport calculations on the systems are performed using the TRANSIESTA package, ${ }^{30}$ which is an ab initio implementation of the nonequilibrium Green's function-density functional theory (NEGF-DFT) transport formalism. ${ }^{30,33}$ We use fully relaxed coordinates for the transport calculation, with the mesh cutoff reduced to $150 \mathrm{Ry}$ and only single- $\zeta$ and polarization (SZP) orbitals in the basis sets. Aside from these settings, the computational parameters for the TRANSIESTA calculations are otherwise the same as those for the SIESTA calculations. We have tested that the choice of the basis set does not appreciably impact our results by calculating some of the structures using the DZP basis set.

To characterize the transport properties of the GNRs, the transmission function around the Fermi energy and at zero bias was calculated. The transmission function is defined by

$$
T(\omega)=\operatorname{Tr}\left\{\Gamma_{L}(\omega) G^{a}(\omega) \Gamma_{R}(\omega) G^{r}(\omega)\right\},
$$

where $\Gamma_{\mathrm{L} / \mathrm{R}}$ are the left/right lead couplings given by twice the imaginary part of the lead self-energy terms, and $G^{r / a}$ are the retarded/advanced Green's functions for the central region. The transmission function is physically representative of the linear response conductance, though in practice doping via either a gate electric field, by chemical means, or with a bias voltage may change the transmission function from its calculated zero-bias form. At low bias or doping, however, the transmission function should give a reasonably accurate representation of the transport properties.

\section{BOND DISSOCIATION ENERGY}

Table I shows the calculated oxygen BDEs for the studied systems in the case of three different GNR widths per system. The zigzag edge has a higher value of the oxygen BDE in comparison to the armchair edge. This edge dependence has been previously reported ${ }^{34}$ and is related to the ZGNR edge states producing a sharp peak in the density of states (DOS) at the Fermi level. The ZGNR edge states participate in chemical bonding and thus contribute to the relative increase of the BDE. Disorder in the form of the protrusion suppresses ZGNR edge states and reduces the DOS at the Fermi level, therefore also decreasing the BDE. The BDE is fairly constant as a function of the ZGNR width for both the protruded and the ideal ribbon.

For ideal AGNRs, the lack of reactive edge states leads to the relative decrease in the oxygen BDE when compared to ideal ZGNRs. The electronic structures divide AGNRs of

TABLE I. Oxygen bond dissociation energies in $\mathrm{eV}$ for the studied geometries in Fig. 1 with ribbon widths of 5, 6, and 7.

\begin{tabular}{lccc}
\hline \hline Width & 5 & 6 & 7 \\
\hline AGNR & 7.31 & 6.93 & 7.08 \\
ZGNR & 7.74 & 7.75 & 7.74 \\
AGNR-prot & 7.10 & 7.46 & 7.30 \\
ZGNR-prot & 6.72 & 6.74 & 6.76 \\
\hline \hline
\end{tabular}


different widths into three different families; AGNRs of width $3 n+2$ (where $n$ is an integer) have the smallest band gaps, whereas $3 n$-AGNRs and $3 n+1$-AGNRs, have increasing band gaps, respectively. ${ }^{17}$ This family structure is clearly evident also in the oxygen BDE values in Table I, where the largest BDE is found for 5-AGNR followed by 7-AGNR and then 6-AGNR. The family structure in the BDE reflects the variations in the DOS available for chemical bonding close to the Fermi energy, or, alternatively, it can be understood on the basis of Clar's theory for aromatic sextets. ${ }^{35}$ Clar's theory indicates that the 6-AGNR with a fully benzenoid structure should be chemically the most inactive, while the 5-AGNR with an incomplete benzenoid structure will be the most reactive.

Adding the protrusion increases the BDE for 6- and 7-AGNRs, but decreases it for the 5-AGNR. This can be understood by the effect of the protrusion, which locally modifies the width of the ribbon to be that of the next family in the AGNR series.

\section{ARMCHAIR RIBBONS}

The transmission functions for an ideal 5-AGNR, both with and without an adsorbed oxygen atom, are shown in Fig. 2. The marked difference in the results with and without oxygen adsorption demonstrates the strong impact that oxygen edge adsorption has on the transport properties of the ideal AGNR. The large depletion in the transmission below the Fermi level, is due to the Fano antiresonance effect, ${ }^{36-38}$ which results from the interference of the localized states (here, the oxygen atomic orbitals) with the continuum states of the ribbon. This understanding is further corroborated by the increase in the oxygen partial density of states (PDOS) that coincides with the decrease in the transmission function (bottom panel in Fig. 2). Here the PDOS is calculated from the retarded Green's function so that it is only nonzero outside the band gap of the ideal nanoribbon leads.

Figure 3 shows the transmission functions for 6-, 7-, and 8-AGNRs. The results for the 5-AGNR system differ qualitatively from the 6- and 7-AGNR cases (Fig. 2). The

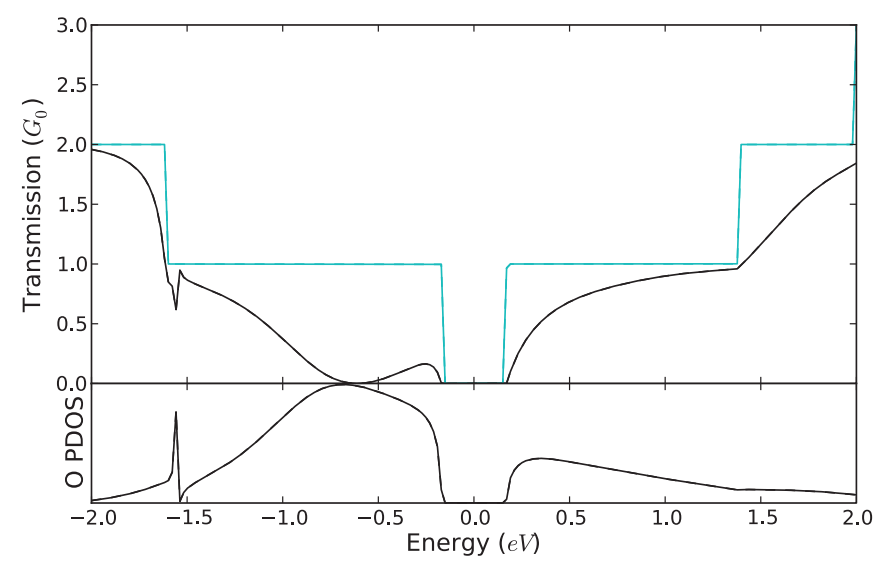

FIG. 2. (Color online) Transmission functions for a 5-AGNR. Black and gray (cyan) curves denote the transmissions for systems with an oxygen atom and with hydrogen termination only, respectively. The bottom panel gives the oxygen PDOS.

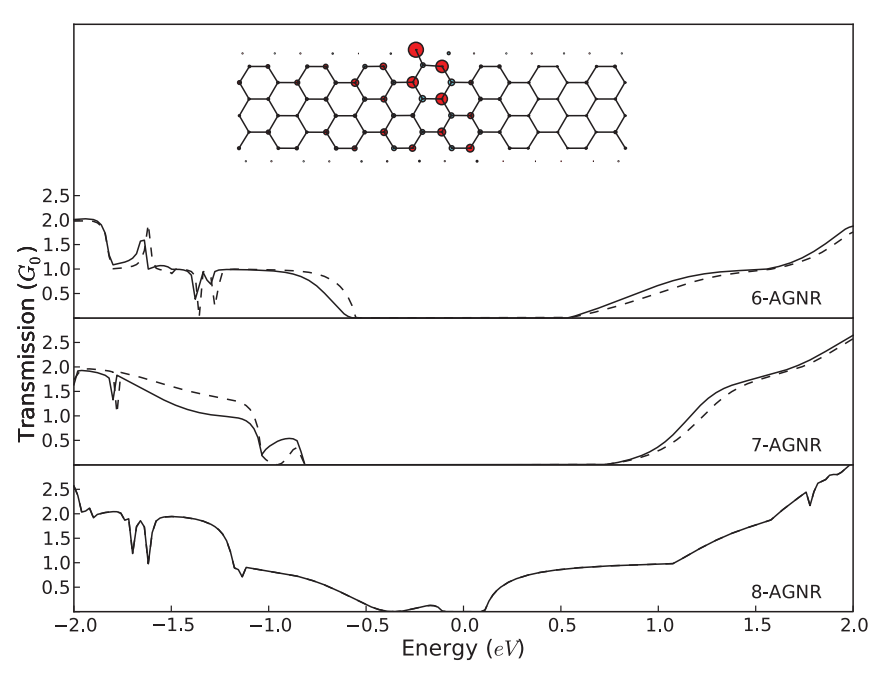

FIG. 3. (Color online) Transmission functions for 6-, 7-, and 8-AGNRs with one adsorbed oxygen atom. The solid curve denotes spin up transmission, and the dashed curve denotes spin down transmission. The inset shows spin up minus spin down Mulliken charges for the 6-AGNR with positive and negative values indicated by dark (red) and gray (cyan) solid circles, respectively.

most notable difference is the appearance of spin polarization localized on the oxygen adsorbate and decaying into the graphene lattice in a manner similar to the decay of the ZGNR edge state. ${ }^{17}$ The magnetic structure is shown in the inset in Fig. 3, where the Mulliken charges (electron charge projected on the atomic orbitals) for the spin up minus spin down charge density are plotted for the 6-AGNR. Similar magnetic structures are also found in the case of the 7-AGNR. In both cases, the localized moments lead to weakly spin-polarized transmission functions.

The reason for the width-dependent properties of AGNRs lies in the AGNR family structure, as previously discussed with respect to the BDE results. An oxygen atom adsorbed onto the edge of the 8-AGNR, which belongs to the same family as the 5-AGNR, does not induce a local magnetic moment and the transmission is therefore very close to that of the oxygen-5-AGNR system in Fig. 2. The appearance of spin polarization in the case of the $3 n$ and $3 n+1$ families can be understood on the basis of the details of the ideal AGNR band structures; the bands of the $3 n$ and $3 n+1$ families, showing relatively wide band gaps, are flatter close to the Fermi energy than those of the $3 n+2$ family, which have nearly vanishing band gaps. The flat bands lead to a high DOS just above and below the Fermi energy, which favors magnetization due to the oxygen states and which contribute to the DOS peak below the Fermi energy.

Figure 4 shows the transmission functions for a protruded 5-AGNR, both with and without an adsorbed oxygen atom. The protrusion itself causes only minor perturbations to the ideal AGNR result (cf. Fig. 2). The protrusion and the oxygen atom together, however, lead to spin-polarized transmission, which is particularly marked below the Fermi energy (Fig. 4). The oxygen-induced dip in the transmission is seen in one spin channel only due to the band gap of the ideal nanoribbon lead. The peak in the spin up oxygen PDOS coincides with the full suppression of the corresponding transmission spin channel. 


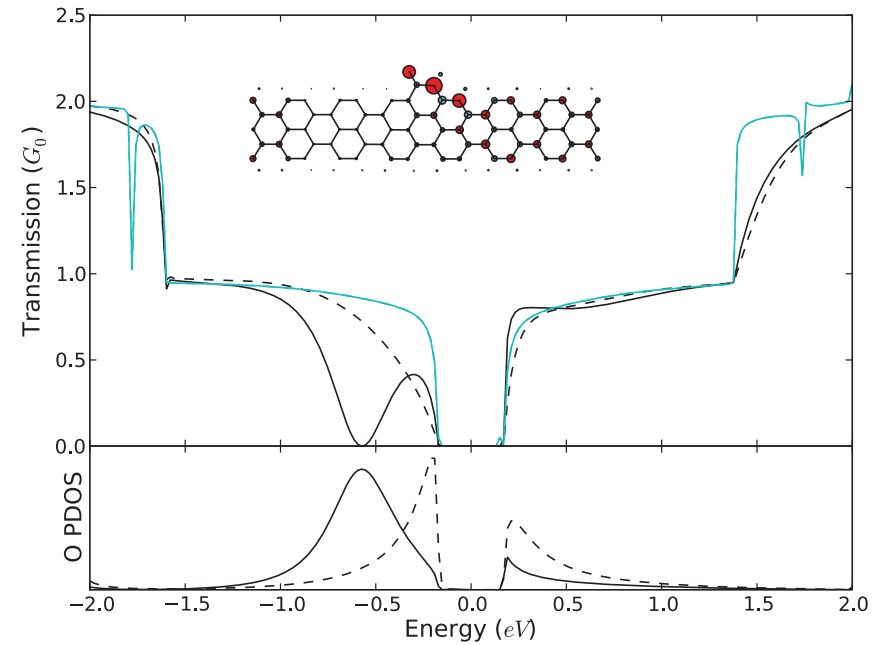

FIG. 4. (Color online) Transmission functions for a 5-AGNR with a protrusion. Black and gray (cyan) curves denote the transmissions for systems with an adsorbed oxygen atom and with hydrogen termination only, respectively. The solid curve denotes spin up transmission and the dashed curve denotes spin down transmission. The inset shows spin up minus spin down Mulliken charges with positive and negative values indicated by dark (red) and gray (cyan) solid circles, respectively.

The peak in the spin down PDOS is cut off by the band gap and thus has limited impact on the transmission. Comparison of Figs. 2 and 4 demonstrates the importance of the precise bonding geometry for the adsorbed oxygen atom and how this markedly affects the transmission results. In contrast to the ideal AGNR (inset of Fig. 3), the local spin polarization in Fig. 4 is mostly limited to the short stretch of zigzag edge at the protrusion, and thus there is little spin polarization inside the bulk lattice.

In Fig. 5 the transmission functions for wider, protruded AGNRs that have an adsorbed oxygen atom are shown. In contrast to the ideal AGNRs, the protruded systems exhibit spin-polarized transmission at all widths, regardless of the

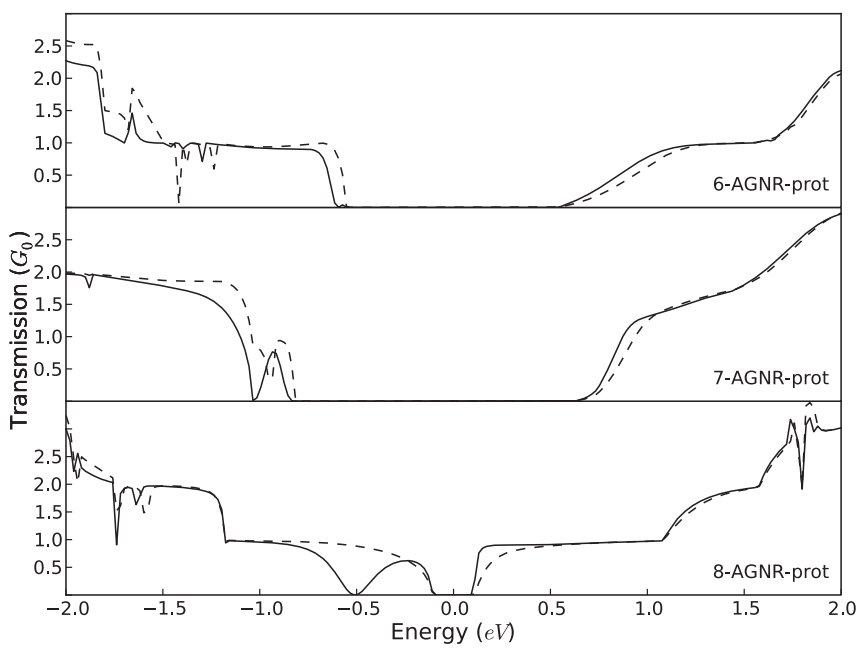

FIG. 5. Transmission functions for protruded 6-, 7-, and 8AGNRs with an adsorbed oxygen atom. The solid curve denotes spin up transmission and the dashed curve denotes spin down transmission.

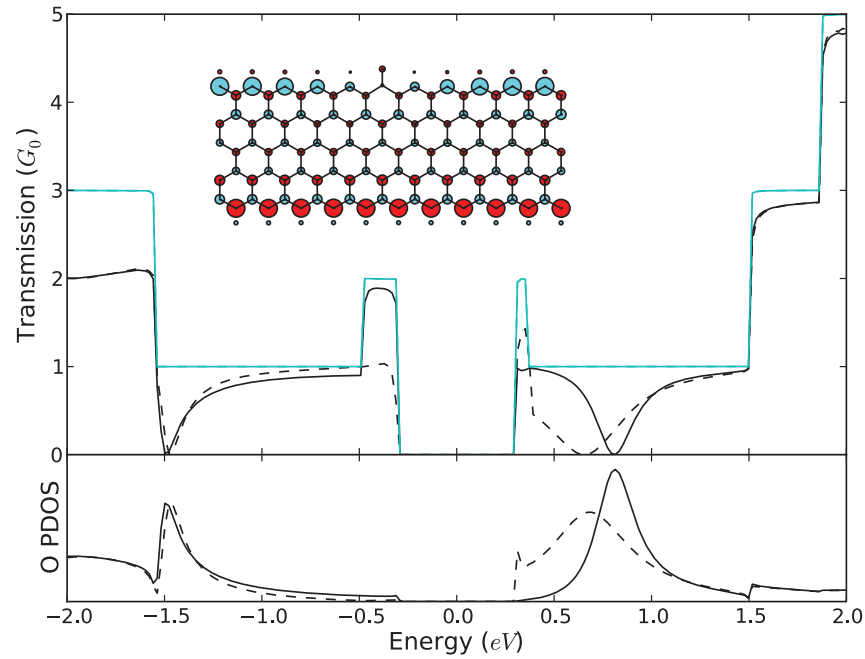

FIG. 6. (Color online) Transmission functions for a 5-ZGNR. Black and gray (cyan) curves denote the transmissions for systems with an adsorbed oxygen atom and with hydrogen termination only, respectively. The solid curve denotes spin up transmission, and the dashed curve denotes spin down transmission. The inset shows the spin up minus spin down Mulliken charges with positive and negative values indicated by dark (red) and gray (cyan) solid circles, respectively. The bottom panel gives the oxygen PDOS.

AGNR family. The AGNR family structure again leads to differences between the different families as can be seen by comparing the panels in Fig. 5. Systems belonging to the same family, however, demonstrate a remarkable similarity in the transmission results, which can be seen by comparing the transmission functions for the oxygenated protruded 8-AGNR in the bottom panel of Fig. 5 with those for the same family 5-AGNR in Fig. 4.

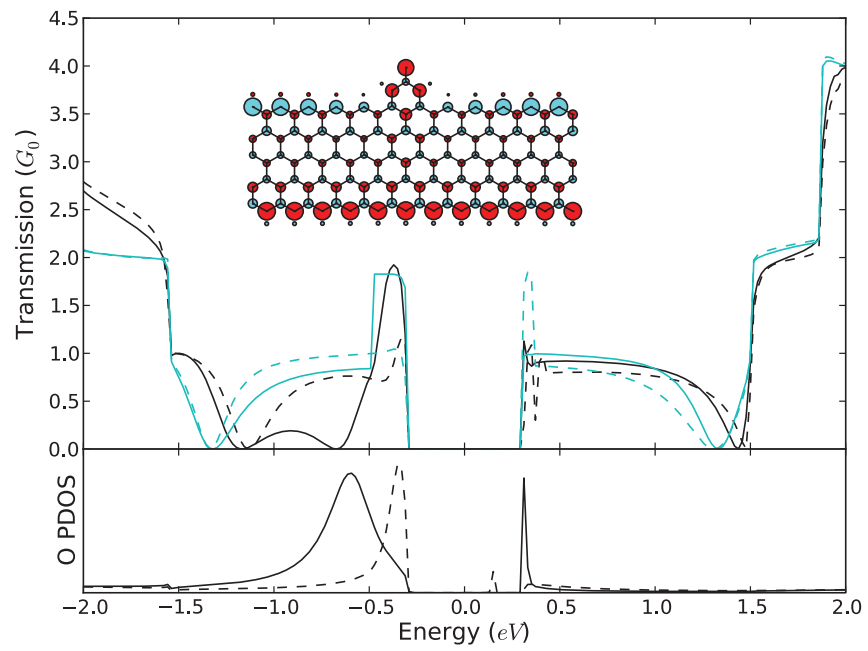

FIG. 7. (Color online) Transmission functions for a protruded 5-ZGNR. Black and gray (cyan) curves denote the transmissions for systems with an adsorbed oxygen atom and with hydrogen termination only, respectively. The solid curve denotes spin up transmission, and the dashed curve denotes spin down transmission. The inset shows the spin up minus spin down Mulliken charges with positive and negative values indicated by dark (red) and gray (cyan) solid circles, respectively. The bottom panel gives the oxygen PDOS. 


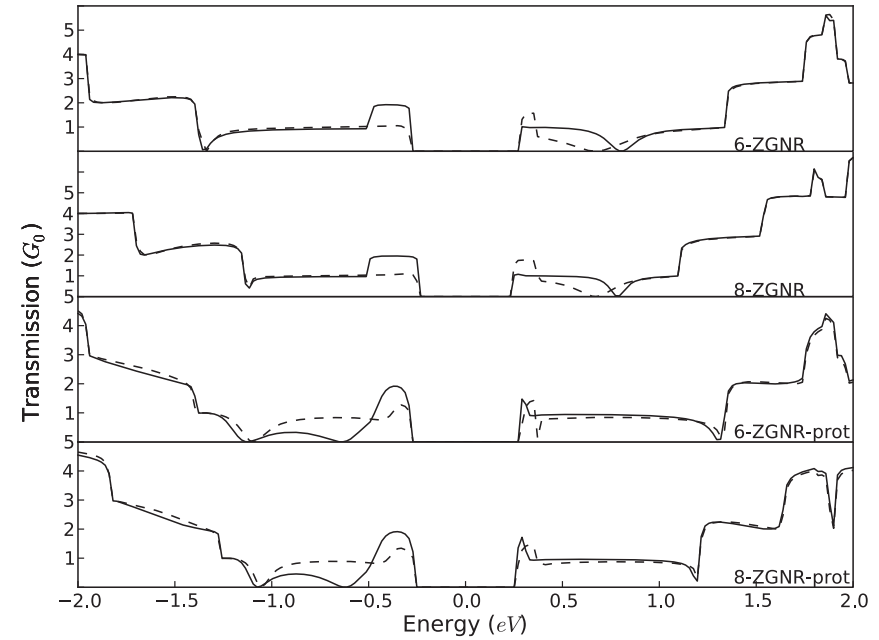

FIG. 8. Transmission functions for 6- and 8-ZGNRs with and without protrusions and with an adsorbed oxygen atom. The solid curve denotes spin up transmission and the dashed curve denotes spin down transmission.

\section{ZIGZAG RIBBONS}

The transmission function for an ideal 5-ZGNR, both with and without an adsorbed oxygen atom is shown in Fig. 6 . The Mulliken charge plot in the inset shows how the oxygen atom suppresses the spin-polarized edge states of the zigzag ribbon. The Fano antiresonance explanation remains valid, as can be seen by the close correspondence of the dips in transmission with the peaks in the oxygen PDOS.

Figure 7 shows the transmission functions for a protruded 5-ZGNR, both with and without an adsorbed oxygen atom. The correspondence between the oxygen PDOS and the transmission function is now significantly weaker. By comparing the transmissions both with and without the oxygen atom, it can be seen that the protrusion alone is the dominant factor that affects the transmission results. When the oxygen atom is added to the protrusion, the locations of the transmission dips are only slightly shifted. This difference with respect to the other systems studied in this work can be understood by recalling the importance of the ZGNR edge states for transport near the Fermi energy. The protrusion, or in general any edge disorder, is enough to suppress the edge states, after which the effect of adding an oxygen atom becomes relatively small. This means that the proposed use of ZGNRs as chemical sensors ${ }^{39}$ may not be possible due to the geometric disorder in real nanoribbon systems.

For zigzag ribbons there is no separation into different families, as there is for the armchair case and thus with the increased ribbon width the transmission functions remain qualitatively very similar. This is evident from Fig. 8 where the results for an oxygen atom adsorbed onto both ideal and protruded 6- and 8-ZGNRs are shown. In comparison with the 5-ZGNR results in Fig. 7, those for the wider ribbons exhibit transmission dips at slightly different locations and the antiresonance peaks are also narrower but otherwise the main features remain the same.

\section{CONCLUSIONS}

We have studied spin-dependent electron transport in GNRs with structural and chemical disorder. The transport properties are found to be affected by edge disorder, particularly via the introduction of Fano antiresonances on the transmission function. For AGNRs, structural edge disorder remains relatively unimportant, whereas the chemical disorder of the oxygen adsorbate dominates the transmission characteristics. For the ZGNRs, structural edge disorder is the most important factor due to the perturbation of the edge states and the adsorbate has a minimal impact on the transport. The transmission properties do not depend largely on the ribbon width for ZGNRs while for AGNRs the threefold family structure has a large impact. The transmissions for AGNRs of different width but within the same family are very similar, resembling the case for ZGNRs. Comparing different families, however, shows large differences in the transmission results and even in the spin distributions.

\section{ACKNOWLEDGMENTS}

This work was performed at COMP, a Center of Excellence of the Academy of Finland for 2006-2011. Funding was also provided by the Nokia Nanosystems collaboration. Computational resources were provided by CSC-IT Center for Science Ltd. A.P.J. is grateful to the FiDiPro program of the Academy of Finland. We thank Mads Brandbyge and Joachim Fürst for useful discussions. *ksaloriu@tkk.fi

${ }^{1}$ K. S. Novoselov, A. K. Geim, S. V. Morozov, D. Jiang, Y. Zhang, S. V. Dubonos, I. V. Grigorieva, and A. A. Firsov, Science 306, 666 (2004).

${ }^{2}$ M. Y. Han, B. Ozyilmaz, Y. Zhang, and P. Kim, Phys. Rev. Lett. 98, 206805 (2007).

${ }^{3}$ J. Bai, X. Duan, and Y. Huang, Nano Lett. 9, 2083 (2009)

${ }^{4}$ F. Schedin, A. K. Geim, S. V. Morozov, E. W. Hill, P. Blake, M. I. Katsnelson, and K. S. Novoselov, Nat. Mat. 6, 652 (2007).

${ }^{5}$ N. Tombros, C. Jozsa, M. Popinciuc, H. T. Jonkman, and B. J. van Wees, Nature (London) 448, 571 (2007).
${ }^{6}$ M. Y. Han, J. C. Brant, and P. Kim, Phys. Rev. Lett. 104, 56801 (2010).

${ }^{7}$ X. Li, X. Wang, L. Zhang, S. Lee, and H. Dai, Science 319, 1229 (2008).

${ }^{8}$ J. Cai, P. Ruffieux, R. Jaafar, M. Bieri, T. Braun, S. Blankenburg, M. Muoth, A. P. Seitsonen, M. Saleh, X. Feng, K. Mullen, and R. Fasel, Nature (London) 466, 470 (2010).

${ }^{9}$ L. Jiao, L. Zhang, X. Wang, G. Diankov, and H. Dai, Nature (London) 458, 877 (2009).

${ }^{10}$ D. V. Kosynkin, A. L. Higginbotham, A. Sinitskii, J. R. Lomeda, A. Dimiev, B. K. Price, and J. M. Tour, Nature (London) 458, 872 (2009). 
${ }^{11}$ T. Shimizu, J. Haruyama, D. C. Marcano, D. V. Kosinkin, J. M. Tour, K. Hirose, and K. Suenaga, Nat. Nanotechnol. 6, 45 (2010).

${ }^{12}$ Y. Kobayashi, K. I. Fukui, T. Enoki, and K. Kusakabe, Phys. Rev. B 73, 125415 (2006).

${ }^{13}$ C. O. Girit, J. C. Meyer, R. Erni, M. D. Rossell, C. Kisielowski, L. Yang, C.-H. Park, M. F. Crommie, M. L. Cohen, S. G. Louie, and A. Zettl, Science 323, 1705 (2009).

${ }^{14}$ X. Jia, M. Hofmann, V. Meunier, B. G. Sumpter, J. CamposDelgado, J. M. Romo-Herrera, H. Son, Y.-P. Hsieh, A. Reina, J. Kong, M. Terrones, and M. S. Dresselhaus, Science 323, 1701 (2009).

${ }^{15}$ M. Engelund, J. A. Fürst, A.-P. Jauho, and M. Brandbyge, Phys. Rev. Lett. 104, 36807 (2010).

${ }^{16}$ M. Fujita, K. Wakabayashi, K. Nakada, and K. Kusakabe, J. Phys. Soc. Jpn. 65, 1920 (1996).

${ }^{17}$ Y.-W. Son, M. L. Cohen, and S. G. Louie, Phys. Rev. Lett. 97, 216803 (2006).

${ }^{18}$ S. Okada, Phys. Rev. B 77, 41408 (2008).

${ }^{19}$ P. Koskinen, S. Malola, and H. Häkkinen, Phys. Rev. Lett. 101, 115502 (2008).

${ }^{20}$ T. Wassmann, A. P. Seitsonen, A. M. Saitta, M. Lazzeri, and F. Mauri, Phys. Rev. Lett. 101, 96402 (2008).

${ }^{21}$ Y.-M. Lin, V. Perebeinos, Z. Chen, and P. Avouris, Phys. Rev. B 78, 161409 (2008).

${ }^{22}$ C. Stampfer, J. Güttinger, S. Hellmüller, F. Molitor, K. Ensslin, and T. Ihn, Phys. Rev. Lett. 102, 56403 (2009).

${ }^{23}$ M. Evaldsson, I. V. Zozoulenko, H. Xu, and T. Heinzel, Phys. Rev. B 78, 161407 (2008).
${ }^{24}$ F. Sols, F. Guinea, and A. H. Castro Neto, Phys. Rev. Lett. 99, 166803 (2007)

${ }^{25}$ S. M.-M. Dubois, A. Lopez-Bezanilla, A. Cresti, F. Triozon, B. Biel, J.-C. Charlier, and S. Roche, ACS Nano 4, 1971 (2010).

${ }^{26}$ F. Cervantes-Sodi, G. Csányi, S. Piscanec, and A. C. Ferrari, Phys. Rev. B 77, 165427 (2008).

${ }^{27}$ G. Lee and K. Cho, Phys. Rev. B 79, 165440 (2009).

${ }^{28}$ X. H. Zheng, I. Rungger, Z. Zeng, and S. Sanvito, Phys. Rev. B 80, 235426 (2009).

${ }^{29}$ J. M. Soler, E. Artacho, J. D. Gale, A. García, J. Junquera, P. Ordejón, and D. Sánchez-Portal, J. Phys. Condens. Matter 14, 2745 (2002).

${ }^{30}$ M. Brandbyge, J.-L. Mozos, P. Ordejón, J. Taylor, and K. Stokbro, Phys. Rev. B 65, 165401 (2002).

${ }^{31}$ J. P. Perdew, K. Burke, and M. Ernzerhof, Phys. Rev. Lett. 77, 3865 (1996).

${ }^{32}$ S. F. Boys and F. Bernardi, Mol. Phys. 19, 553 (1970).

${ }^{33}$ H. Haug and A.-P. Jauho, Quantum Kinetics in Transport and Optics of Semiconductors, 2nd ed., Springer Series in Solid State Sciences, Vol. 123 (Springer-Verlag, Berlin, 2008).

${ }^{34}$ D.-E. Jiang, B. G. Sumpter, and S. Dai, J. Chem. Phys. 126, 134701 (2007).

${ }^{35}$ M. Baldoni, A. Sgamellotti, and F. Mercuri, Chem. Phys. Lett. 464, 202 (2008).

${ }^{36}$ U. Fano, Phys. Rev. 124, 1866 (1961).

${ }^{37}$ J. U. Nöckel and A. D. Stone, Phys. Rev. B 50, 17415 (1994).

${ }^{38}$ J. A. Fürst, M. Brandbyge, A.-P. Jauho, and K. Stokbro, Phys. Rev. B 78, 195405 (2008).

${ }^{39}$ B. Huang, Z. Li, Z. Liu, G. Zhou, S. Hao, J. Wu, B.-L. Gu, and W. Duan, J. Phys. Chem. C 112, 13442 (2008). 